\title{
Etymologische Untersuchungen über diejenigen Namen der chemischen Elemente, welche ihren internationalen ond nationalen Sigeln*) za Grunde liegen, mit besonderer Berücksichtigung ihrer deutschen Benennungen ${ }^{1}$ );
}

von

\section{Paul Diergart.}

Die Ansichten über die chemischen Elemente haben im Laufe der Zeit sehr gewechselt. Ihrer Benennung liegen wesentlich gleiche und ähnliche Beziehungen zu Grunde.

Die heutigen Elemente sind benannt worden

1. nach der Eigenschaft:

a) im Elementarzustand:

a) physikalisch: argentum, aurum, Gold, Blei, plumbum, Eisen, platina, chlorum, iodum, phosphorus, radium, stannum, ferrum, hydrargyrum, Quecksilber, Zink, zineum.

B) physiologisch: bromum, Stickstoff, azotum.

r) chemisch: nitrogenium, oxygenium, Sauerstoff, Wasserstoff, hydrogenium, argon.

b) im Verbindungszustand:

a) physiologisch: Schwefel, glucinium, osmium.

1) Die vorliegende Arbeit ist veranlasst worden durch die Abhandlung des Herrn Dr. Berendes über "Die Namen der Elemente" (Chem.Ztg. 1899, Nr. 11), welche einer wissenschaftlichen etymologischen Grundlage entbehrt und nur eine äusserliche Zusammenstellung der bekanntesten Erklärungen nach der Buchstabenfolge darbietet. Ich werde daher bei Besprechung der einzelnen Benennungen wiederholt auf die genannte Arbeit, sowie anf diejenige des Herrn Dr. Freuden berger zurückkommen müssen, welcher derselhen eine auch nicht einwandfreie Erwiderung hat zu Theil werden lassen. - Am Schlusse befindet sich das Verzeichniss der Abkürzungen und der benutzten Quellen.

*) Ich habe den Ausdruck Sigel statt Zeichen gebraucht für die zur Bezeichnung der Elemente gewählten Buchstaben, um jeđe Verwechselung mit der alchimistíschen Bezeichnungsweise durch Kreise, Hallomonde u. s. w. auszuschliessen.

Journal 8. prakt. Chemie [2] Bd. 61. 
498 Diergart: Etymologische Untersuchungen u.s. w.

B) physikalisch: chromum, iridium, rhodium, praseodym, thallium, indium, caesium, rubidium.

2. nach der Art und Weise ihrer Entdeckung: lithium, tellurium, helium, lanthanium, kryptonium, dysprosium, decipium, neon, neodym, xenon.

3. nach Stoffen, in denen sie vorkommen: arsenum, antimon, stibium; molybdaenum, borum, zirconium, carbonium, Kohlenstoff, fluor, barium, natrium, kalium; aluminium, silicium, calcium, Wolfram. samarium, holmium, cadmium. manganium, magnesium, beryllium, strontium, yttrium, erbium, terbium; ytterbium.

4. nach dem Orte des Vorkommens: cuprum, Kupfer, ruthenium, scandium, thulium, germanium, gallium, polonium. Wismuth, bismutum.

5. nach mythischen und sagenhaften Personen: vanadium, thorium, titanium, uranium, cerium, palladium; tantalum, niobium.

6. sinnbildlich: selenum, Kobalt, cobaltum, Nickel, niccolum.

7. nach Forschern: gadolinium, philippium, mosandrium.

8. nach keinem der angeführten Gesichtspunkte lassen sich behandeln: Zinn, Silber, sulfur.

Die aristotelisch-naturphilosophische Anschauung von der Vierzahl der Elemente ${ }^{1}$ ) ist fast 2000 Jahre im grössten Ansehen geblieben und noch in der scholastischen Philosophie der Abendländer bis ins 16. Jahrhundert hinein grundlegend gewesen. Erst dann hat sie Abänderungen nach Art und Zahl der Elemente erfahren.

Boyle hat als erster die Frage nach den Elementen in dem Sinne aufgefasst, wie sie noch jetzt wenigstens grundsätzlich behandelt wird, nämlich nur die für die Chemie nicht weiter theilbaren Bestandtheile der Materie Elemente zu nennen. Solcher Elemente sind nach der neuesten Forschung ungefähr 75 (gegen 64 i. J. 1863) bekannt. Ihr Vorhandensein sowohl

1) Vierzahl der Elemente nach der pythagoräischen Vorliebe für die heilige Tetraktys, s. Hermann Diels, Elementum, Leipzig 1899. 
Diergart: Etymologische Untersuchungen u.s.w. 499

wie ihre weitere Theilbarkeit, namentlich in Bezug auf einige seltenere wird von maassgebender Seite sehr in Frage gestellt.

Der Benennung dessen, was man Elemente genannt hat, haben alle Zeit ihre Eigenschaften, sinnbildliche Uebertragungen und Beziehungen anderer Art zu Grunde gelegen. Schon die

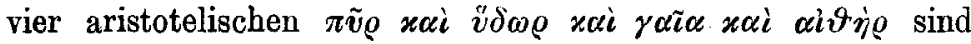
etymologisch hierauf zurückzuführen. Im Einzelnen hierfür den genauen Beweis zu bringen, würde zu weit führen. IIṽ kann nach Pott als Sinnbild der reinigenden Kraft des Feuers hingestellt werden, $\ddot{v} \delta \omega \varrho$ ist das, womit benetzt wird, $\gamma \propto \tilde{i} \alpha-\gamma \tilde{\bar{\eta}}$ kann sinnbildlich als "milchende Kuh" aufgefasst werden, und civì bedeutet wahrscheinlich die durchs Brennen gereinigte, obere reine Luft. $\left.{ }^{1}\right)$ Die tria principia des Basilius Valentinus: mercurius et sulfur et sal, Quecksilber, Schwefel und Salz, und die fünf Principien des Le Fèvre (traité de la chymie 1660): Wasser oder Phlegma, geistiges oder merkurialisches Princip, schwefliges oder öliges Princip, Salz und Erde werden sich grossentheils im Laufe der Ausführung etymologisch auf ähnliche Beziehungen zurückführen lassen. Besonders ist dies der Fall bei der Benennung unserer heutigen Elemente. Denn sie sind, wie ihre etymologische Untersuchung ergiebt, benannt worden nach ihrer Eigenschaft, ferner nach der Art und Weise ihrer Entdeckung sowie nach Stoffen, in denen sie vorkommen. Auch Benennungen nach dem Orte, nach mythischen und sagenhaften Personen, und sinnbildliche Bezeichnungen finden sich gleichfalls neben denen nach verdienstvollen Männern, während einige wenige Benennungen sich nicht nach diesen Gesichtspunkten behandeln lassen.

Bezeichnungen nach der Eigenschaft.

Vor allem anderen sind es die Eigenschaften, die bei der Benennung unserer Elemente die wichtigste Rolle spielen, und zwar diejenigen, welche sie sowohl wie ihre Verbindungen physikalisch und chemisch kennzeichnen.

Das Aussehen, die Farbe, hat dem argentum (Ag), einem der am frühesten bekannten Metalle ${ }^{2}$ ), den Namen gegeben, und

1) S. Curtius, "Grundzüge der griech. Etymologie", Leipzig 1866.

$\left.{ }^{2}\right)$ S. Nachtrag 1 . 
zwar nicht nur in der lateinischen, sondern auch in mehreren der älteren Sprachen. Dies ist namentlich sichtbar in der griechischen Bezeichnung óørvoos.

Nach Curtius und Weise ${ }^{1}$ ) hat das griechische Wort

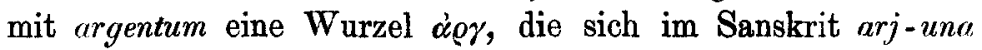
"licht", raj-ata "weiss", (W. arj. raj.) wiederfindet. Aehnlich ist es mit dem vielleicht noch früher bekannten aurum $(\mathrm{Au})$, das wahrscheinlich mit aurora "Morgenröthe" zu einer Wortgruppe gehört. Diesem liegt nach Curtius die Skr.-W. vas, urchati, ,,brennen, leuchten" zu Grunde. "Vas" ist vermuthlich gleich der durch Zulaut entstandenen W. aus (aus-osa, aurora, „Morgenröthe") der europäischen Völker. Den germanischen Völkern ist bei diesem Metalle mehr das Gelbe als das Rothe ins Auge gefallen. Deshalb liegt in ihren Sprachen die W. ghel mit der Urbedeutung, ,gelb sein" zu Grunde. Hiervon ist nach Kluge ${ }^{2}$ ) augenscheinlich unser nhd. "Gold" eine participiale Ableitung. [Skr. hari, harita, "gelb“, hiranya ,Gold", lit. geltas "gelb"]. Das germanische blîwa, ahd. blîo (für blîw) mhd. bli (Gen. blîwes) nhd. „Blei“" ist nach Persson ${ }^{3}$ ) mit dem lit. bleivas „licht, klar" verwandt.

Schon die Alten haben an diesem Metalle die Eigenschaft des Abfärbens wahrgenommen. Deshalb ist es șchon im Skr. bahu-mala eig. "sehr schmutzig" benannt worden. Hieraus ist nach Curtius und Weise das gr. $\mu$ ód vißos und das lat. plumbum (Pb) entstanden. ${ }^{4}$ )

1) „Die griechischen Wörter im Latein“, Leipzig 1882.

9) Kluge, Etymologisches Wörterbuch der deutschen Sprache, Strassburg 1899, 6. Aufl. $19,273$.

3) Bezzenberge's Beiträge zur Kunde der idg. Sprachen, 1877 ff.,

4) Die Aufstellung einer wahrscheinlichen Stammform unterbleibt aus Gründen der Vorsicht. Jedenfalls ist die ursprüngliche Uebereinstimmung von $\mu$ ó $\lambda v \beta o s$ und plumbum zweifellos. $\mu$ ó $\lambda v \beta o s=$ plumbum, das für ein ursprüngliches mlumbum steht. Es bleibe unerörtert, ob der Uebergang von $m$ in $p$ nur Dissimilation ist, oder ob sich erst aus dem Stimmton des $m$ ein $p$, bezw. ein o (also mplu wie Fähn(d)rich entwickelt hat, vor dem nachher $m$ geschwunden ist.

Plumbum hingegen auf eine Onomatopöie zurückzuführen, um die Schwere des Metalles zu bezeichnen, wie Berendes thut, kann keinerlei Unterstützung finden und fällt in sich zusammen. Der technische Ausdruck Onomatopöie ist hier überhaupt nicht am Platze. 
Diergart: Etymologische Untersuchungen u.s. w. 501

Auch das Eisen hat nach Grimm ${ }^{1}$ ) und Kluge vielleicht von seinem Aussehen, bezw. seiner äusseren Aehnlichkeit mit Eis (Glanz) seinen Namen. [got eisarn, ahd. isarn, später isan, mhd. isen, asächs. isarn, anord. jarn für iarn, schwed.dän. jern.] In diesen Formen haftet das $r n$, wälırend das $s$ der ersten Silbe unterdrückt erscheint (vergl. lat. pomum für posmum). „Sie schliessen sich an ans ir. iarran, iarun, welsch. heiarn, arm. houarn, was um so mehr bedeutet, als sich auch zwischen eis und kelt. eirr, eira Einstimmung findet. Für eisarn bleibt also auch die für eis angenommene Wurzel glaublich. Wie der Krystallstein wörtlich aus gefrorenem Wasser entsprang, konnte auch der Glanz des Metalles auf Eis zurückgeführt werden. Abgelegen sind die Ausdrücke urverwandter Sprachen lat. ferrum, ital. ferro, span. hierro, frz. fer, gr.

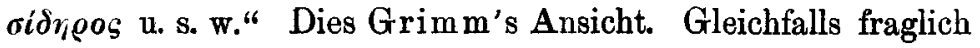
ist nach Kluge Verwandtschaft mit ahd. êr, got. aiz, lat. aes, "Erz"6. - Auch auf eine äussere Aehnlichkeit mit einem anderen Körper zurückzuführen ist die Benennung des Elementes Platina (Pt). In Spanien um die Mitte des 18. Jahrh. wieder der Gegenstand chemischer Untersuchungen, ist es wegen seiner Aehnlichkeit mit dem span. plata = Silber nach dessen Verkleinerungswort platina so genannt worden. Es hat den Beinamen del Pinto, weil man zuerst sein Vorkommen im Goldsande des Flusses Pinto in Neu-Granada beobachtet hat. ${ }^{2}$ ) Die Annahme Platina del Tinto nach Rio del Tinto in Andalusien hat sich nicht bestätigt.

Ebenso wie das Platin ist auch das chlorum (Cl), lange bevor ihm seine jetzige Benennung zu Theil geworden ist, der Gegenstand chemischer Untersuchungen gewesen. Bis zu Anfang unseres Jahrhunderts unter der Bezeichnung "oxydirte Salzsäure" bekannt, ist es in England wegen seiner grünlichgelben ( = $\chi \lambda$ woós) Farbe "Chlorine" oder "chloric gas" genannt worden. In Frankreich hat man es zu chlore abgekürzt, dem dann unsere Benennung "Chlor" entnommen ist. Das ihm chemisch sehr ähnliche iodum (J) Jod dagegen hat von Anfang an, wo darüber Mittheilungen gemacht worden sind, stets diese Bezeichnung gehabt. Erst zu Anfang unseres Jahrhun-

1) Deutsches Wörterbuch, Leipzig 1873.

2) S. Herm. Kopp, „Geschichte der Chemie“, Braunschweig 1843. 
502 Diergart: Etymologische Untersuchungen u.s. w.

derts in Frankreich entdeckt, ist ihm dort dieser Name wegen

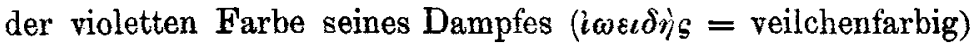
gegeben worden.

Wie bei diesen Elementen das Aussehen bezüglich ihrer Benennung maassgebend gewesen ist, so ist der phosphorus $(\mathrm{P})$ Phosphor durch seine Eigenschaft, im Dunkeln zu leuchten, zu

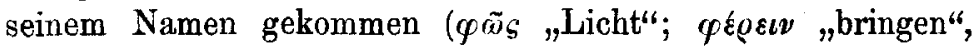
also „Lichtbringer “).

Im vorigen Jahrhundert hat man unter "Phosphor" allgemein jeden Körper verstanden, der diese Eigenschaft zeigt. Insonderheit ist der bononische Leuchtstein (Schwerspat), der bereits im 17. Jahrhundert unter dem Namen Litheosphorus (aus litheophosphorus "steinerner Lichtträger") beschrieben ist, so benannt worden, ferner der in Deutschland hinsichtlich dieser Eigenschaft bekannt gewordene wasserfreie salpetersaure Kalk. Bald hat sich dann der Name auf den jetzt als "Phosphor" bezeichneten Körper beschränkt. Der Eigenschaft, optisch aktive Strahlen auszusenden, verdankt das vor Kurzem gefundene „Radium" seinen Namen, von radius, Stab, Speiche übertr. Strahl.

Nicht minder als die optischen sind die mechanischen Eigenschaften der Elemente bei ihrer Benennung ins Gewicht gefallen. Das lat. stagnum, das nach Vanicek ${ }^{1}$ ) dasselbe ist wie stannum $(\mathrm{Sn})$, geht nach Curtius und Vanicek vielleicht auf die Skr.-Wörter stha "stehen, Standhalten" und sthâvara „fest, beständig“ zurück, über kelt. stean, stan. ${ }^{2}$ ) Stannum wäre somit als der verkörperte Begriff des Festen anzusehen. ${ }^{3}$ )

Vielleicht bedeutet ferrum (Fe) nach Hintner ${ }^{4}$ ) dasselbe. Er bringt es mit fertum in Zusammenhang, dem das skr. dhrti „Festigkeit“ zu Grunde liegen könne. Gerade über ferrum sind die Ansichten der Etymologen sehr verschieden. Nach Vanicek bedeutet es gleichfalls das "Starre", indem er

1) „Etym. Wörterb. der lat. Sprache“. Leipzig 1881.

8) Vgl. Heinrich Leo, „Angelsächsischer Glossar". Halle 1872.

3) Das stannum bei Plini us ist, wie bereits Kopp festgestellt hat, nicht Zinn, sondern wahrscheinlich eine Bleilegirung gewesen. Plin. „(stannum) fit et alio modo, mixtis albi plumbi nigrique (Zinn und Blei) paribus libris." Stannum = Zinn nachweisbar erst seit dem 4. Jahrh.

4) Kleines Wörterb. der lat. Etymologie. Brixen 1873. 
Diergart: Etymologische Untersuchungen u. s. w. 503

es mit skr. harsh "starr, steif werden" zusammenbringt. Weise möchte an semitischen Ursprung glauben, Entlehnung aus hebr. barzel (Eisen) $=$ assyr. parzilla $=$ sumerisch barzal, was sehr wenig einleuchtet.

Einfacher liegen die etymologischen Verhältnisse beim Hydrargyrum (Hg), einer Benennung, die sich zuerst bei Plinius findet. Er versteht darunter das aus Zinnober künstlich dargestellte Quecksilber. Hydrargyrum steht offenbar mit dem gr. zuerst von Dioskorides (1. Jahrh. n. Christus) beschriebenen ígó́gyvos in Verbindung, das nichts anderes

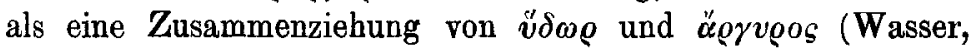
Silber) ist. Plinius bezeichnet das gediegen vorkommende

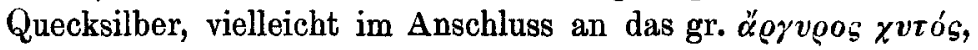
als argentum vivum, von dem unser "Quecksilber" eine einfache Uebersetzung ist. Denn queck ist das nd. quick (engl. quiek, hd. keck) = lebendig, lebhaft. "Quecksilber" ist also lebendiges Silber, d. h. ein in Farbe und Glanz dem Silber ähnliches Metall, das jedoch nicht wie dieses fest, sondern flüssig ist.

Gleichfalls germanischen Ursprungs ist jedenfalls unser erst nhd. "Zink". Es kommt zuerst vor bei Basilius Valentinus im 15. Jahrhundert. In seinem „Triumphwagen des Antimonii" schreibt er: „Es wird auch wohl ohne die" (gleich ausser den) ,gewissen Metalle ein Mineral geboren aus den tribus principiis" (Salz, Schwefel, Quecksilber) "als Vitriol oder anderes mehr, als Cobolt, Zincken - -." Allerdings versteht er hiernach unter dem "Zincken" nicht ein eigentliches Metall. Dass es jedoch dasselbe wie unser metallisches Zink ist, erhellt aus einer Stelle bei Paracelsus. In seinem "Traktat von Mineralien" schreibt er: "also ist noch ein $\mathrm{Me}$ tall, als der Zincken; derselbig ist unbekannt in der Gemeine und ist dermassen ein Metall einer sonderlichen Art, _- -.." Dieser "Zincken" hängt nach Kluge jedenfalls mit "Zinn“ zusammen, an das bei der Entlehnung ins Slavische ein slav. Suffix k getreten, mit dem es als "Zink" wieder ins Deutsche gedrungen wäre. Andere vermuthen Zusammenhang mit ,Zincken", weil das Zinn sich beim Schmelzen in Zincken ansetze. ${ }^{1}$ )

1) Berendes' Ansicht, das lat. cincinnus ,gekräuselt" wegen des blättrig-krystallinischen Bruches zur Erklärung herbeizuziehen, ist hinfällig. 
504 Diergart: Etymologische Untersuchungen u.s. w.

Das dem Sigel $\mathrm{Zn} \mathrm{zu}$ Grunde liegende zincum ist das ins Lateinische übertragene Zink. Es wird in Agricola's „De natura fossilium" augenscheinlich zuerst gebraucht. Dort sagt der Verfasser nach der Besprechung des Galmei (cadmia): "Cum hac cadmia et pyrite cognationem habet mistum, quod Norici et Rheti Zuicum vocant; - - . Zuicum dürfte sicherlich ein Schreibfehler sein statt Zincum, zumal in seinem „Bermannus" die Bezeichnung zincum nochmals vorkommt.

Weniger physikalisch als physiologisch ist die Eigenschaft des bromum (Br) Brom, welche diesem Element den Namen gegeben hat. Wegen seines $\mathrm{ihm}$ eigenen Geruches ist es im Anfange unseres Jahrburderts in Frankreich so benannt worden

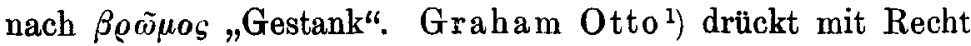
sein Bedauern darüber aus, dass man das Brom nicht wie das Chlor und Jod nach der Farbe seines Gases benannt hat, was in Anbetracht seiner grossen Aehnlichkeit mit Chlor und Jod passender gewesen wäre. Gleichfalls physiologisch ist die Wirkung des Stickstoffs, dessen Ableitung sich von selbst ergiebt. In Frankreich wird dieses Element als azote, azotum (Az) bezeichnet, um gleichfalls an die erstickende Eigenschaft des Gases zu erinnern, aus $\zeta \omega \tau$ เxòs „das Leben erhaltend“ und $\alpha$ privativum.

In der allgemeinen Bezeichnungsart heisst dieses Gas Nitrogenium $(\mathrm{N})$, d. i. ins Lateinische übertragenes nitrogène. Diese Benennung liegt jedoch nicht seiner chemischen Eigenschaft in der belebten Welt wie beim "Stickstoff" und „azote", sondern diejenige in der unbelebten Welt zu Grunde, denn der Name nitrogène ist erst vorgeschlagen worden, nachdem man entdeckt hatte, dass das Gas an der Zusammensetzung der Salpetersäure theilnimmt. Daher aus nitrum "Salpeter" (nicht vic $\varrho o v$ s. unter $\mathrm{Na}$ ) und $\gamma \varepsilon v v \alpha^{\prime}(0=$ bilden.

Ein Bestandtheil sehr vieler Säuren ist das Oxygenium (O), das ins Lateinische übertragene oxygène. Diese Bezeich. nung ist aus dem erwähnten Grunde ( $\dot{o} \xi \grave{v} \varsigma=$ sauer) zuerst in Frankreich eingeführt worden für das unter dem Namen "Dephlogistirte Luft, Feuerluft oder Lebensluft" bekannte Gas. Sie ist im Deutschen durch "Sauerstoff" wiedergegeben

1) „Ausführl. Lehrb. der Chemie“, Braunschweig 1863. 
Diergart: Etymologische Untersuchungen u.s.w. 505

worden, eine Benennung, die irrthümlicher Weise einen sauren, bitteren Geschmack desselben voraussetzen muss. Aehniich zu verstehen ist unsere Benennung "Wasserstoff", der aber nur desbalb so heisst, weil er unter Sauerstoffaufnahme zu Wasser verbrennt. In Frankreich hat man dieses Gas daher hydrogène $(\mathbf{H})$ genannt, etymologisch ähnlich wie ritrogène und oxygène. In der allgemeinen Bezeichnungsweise ist es durchs lat. hydrogenium wiedergegeben worden. $\quad \boldsymbol{v} \dot{\delta} \omega \varrho=$ Wasser, $\gamma \varepsilon v v \alpha^{\prime} \omega=$ bilden). ${ }^{1}$ )

In Bezug auf die chemische Verwandtschaft entgegengesetzte Eigenschaften hat das Element argon (A), was auch bei seiner Benennung berücksichtigt worden ist. "̌orov „Wirkung" und $\alpha$ privativum, also „ohne Wirkung“. (Erst der neuesten Forschung ist es gelungen, Andeutungen dafür zu geben, dass es mit anderen Körpern in Verbindung tritt.)

Bei ihm sowohl wie bei allen bisher behandelten Elementen sind nur die Eigenschaften im Elementar-Zustand in Bezug auf ihre Benennung in Betracht gekommen, und es ist interessant, wie mannigfach auch die Eigenschaften ibrer Verbindungen ihre Benennung herbeigeführt hat.

Zwar chemischer Art, wie zuletzt, sind die in Frage kommenden Eigenschaften nicht, sie neigen mehr in das Gebiet der Physiologie und Physik hinüber. Unser nhd. Ausdruck "Schwefel" ist nach Kluge ohne Zweifel ein gemeingermanisches Wort: ndl. zweafel, angls. swef, schw. swafvel, got. swibls, Schwefel. Kluge sagt weiter darüber: „Wenn das agerm. sweblaz „Schwefel“ nicht uralte Entlehnung aus irgend welcher unbekannten Quelle ist, darf man vielleicht an $\mathrm{Zu}$ gehörigkeit zu der altindog. W. swep "schlafen" denken. (Skr.

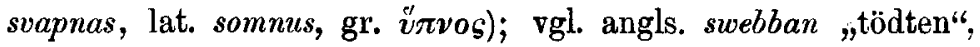
anord. swalfa „tödten, einschläfern“. „Schwefel" ist also eig.

1) Ea ist ausgeschlossen, die Benennung „Wasserstoff" dem Umstande zuzuschreiben, dass das Gas aus dem Wasser dargestellt worden ist. Nach Kopp, Bd. 3, S. 262, ist die Bezeichnung hydrogen oder Wasserstoff i. J. 1787 in der antiphlogistischen Bezeichnungsweise sufgestelit worden. 1783 war entdeckt worden, dass sein Verbrennungsprodukt Wasser sei, nachdem man schon 1776 die Bildung von Wassertropfen beim Verbrennen des Gases beobachtet hatte. Erst 1789 ist der Wasserstoff aus dem Wasser hergestellt worden (Kopp, Bd. 3, S. 274). 
506 Diergart: Etymologische Untersuchungen u.s. W.

„einschläfernder, tödtender Stoff" ", vom giftigen Dampfe des brennenden Schwefels $\left(\mathrm{SO}_{2}\right)$.

Das in Frankreich und England unter dem Sigel G (Glucinium. Glucinum) geführte Element Beryllium, von dem später die Rede sein wird, hat diese Benennung nach dem süssen (= $\gamma \lambda v x v_{\text {ş }}$ ) Geschmack seiner Salze. Auf den eigenartigen Geruch des flüchtigen Osmiumoxyds ist die Benennung des Elementes osmium (Os) von ó $\sigma \mu \eta^{\prime}=$ „Geruch ", ó $\sigma \mu \varepsilon \tilde{\imath} \nu$ ,riechen" zurückgeführt worden.

Noch weniger physiologisch als vielmehr physikalisch sind die Eigenschaften, die der Benennung derjenigen Elemente zu Grunde liegen, deren Salze sich durch schöne Färbung auszeichnen. Vor allen sind es die Chrom- und Iridiumsalze, denen eine prachtvolle Verschiedenheit der Farbe eigen ist.

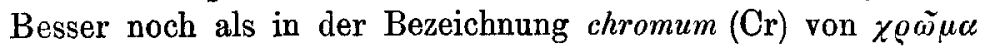
„Farbe" ist dies im Namen iridium (Ir) wiedergegeben worden, die dem gr. iocs = „Regenbogen" entnommen ist. Weniger verschieden in der Farbe sind die Salzlösungen des Elementes rhodium (Rh). Der Entdecker hat diesen Namen gewählt vom

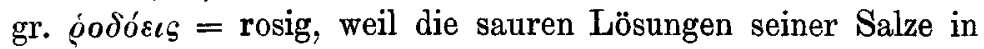
der Regel rosenroth sind. Die lauchgrüne Farbe der Praseodym $(\mathrm{Pr})$-Salze hat diesem Element vom gr. $\pi \varrho \dot{c} \sigma o v=$ Lauch den Namen gegeben. Es ist das lauchgrüne Dym, als Bestandtheil des früher als Element aufgefassten Didyms, von $\delta i \delta v \mu o \iota=Z_{\text {willinge, }}$ weil sich dies stets in Gesellschaft der später zu besprechenden Elemente Cer und Lanthan findet.

Hierher gehören auch die Elemente, die spectral-analytisch entdeckt und nach ihren Hauptspectrallinien benannt worden sind, die ihre Salze und auch theils sie selbst im Bunsenschen Spectralapparat liefern. Daher der Name des Elementes

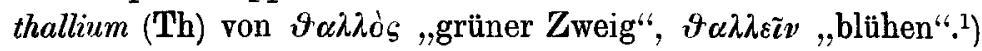

Ebenso hat das Element Indium oder Indigum (In) nach der indigoblauen Linie seinen Namen. Die Heimath der blauen Indigopflanze ist Indien, ihre Benennung daher örtlich. Gleichfalls blau, jedoch heller als die des In ist die Hauptlinie des caesium $(\mathrm{Cs})$ von caesius ,bläulichgrau“".

1) Seine Benennung nach Berendes dem Umstande zuzuschreiben, dass es als "Zweig, Schössling:" in dem Flugstaube des gerösteten Schwefelkieses entdeckt worden ist, beruht auf einem Irrthum. 
Diergart: Etymologische Untersuchungen u.s. w. 507

Die dunkelrothe Linie (rubidus $=$ dunkelroth) hat dem Element rubidium (Rb) seinen Namen gegeben.

\section{Benennungen nach der Art und Weise der Entdeckung.}

Ein noch schöneres Roth liefert das Lithiu m (Li), dessen Benennung jedoch nicht diesem Umstande zuzuschreiben ist. Sie ist vielmehr auf die Art und Weise seines Vorkommens zurückzuführen. Nachdem der Entdecker die alkalische Eigenschaft des Elementes festgestellt hatte, hat er es als Begleiter von Kali und Natron vergeblich in Pflanzenaschen gesucht, hat es vielmehr nur in Mineralien gefunden. Daher hat er seine ausschliessliche Zugehörigkeit zum Mineralreiche angenommen und ihm nach dem gr. $\lambda i \vartheta o s$, ,Stein", $\lambda i \vartheta \varepsilon \iota s$ „,steinern" den erwähnten Namen gegeben. Nachträglich ist es auch in Aschen von Pflanzen, z. B. der Runkelrübe, gefunden worden.

Ganz ähnlich liegen die etymologischen Verhältnisse beim Element tellurium (Te), das in Bezug auf sein gediegenes Vorkommen in der Erde (= tellus) so benannt worden ist. Das Gegentheilige besagt der Name des Elementes helium (He) vom gr. "̈גıos „Sonne“. Denn dieses Element ist mittelst des Spectral-Apparates zuerst auf der Sonne und später erst auf der Erde entdeckt worden, wo es zu den seltensten und schwer zugänglichsten Stoffen gehört.

Der Begriff des Seltenen und schwer Zugänglichen ist auch bei der Benennung mehrerer, insonderheit der neuesten und noch fraglichen Elemente maassgebend gewesen. So sind die Namen der Elemente lanthanium (La) und kryptonium (Kr) vom gr. $\lambda \alpha \nu \vartheta \alpha^{\prime} \nu \varepsilon \iota \nu$, bezw. $x \varrho u ́ \pi \tau \varepsilon \iota \nu$,verborgen sein, verbergen" abzuleiten, weil sie sich so lange dem Auge des Entdeckers

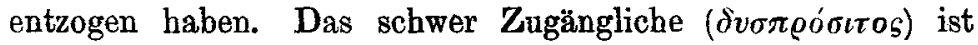
das Dysprosium (Dy). Dasjenige, welches den Entdecker irre geführt hat, ist das Decipium (Dp) von decipere, eig. ,wegfangen“" übertr. „,bethören, durch den Schein betrügen, täuschen, irre leiten". Neon $(\mathrm{Ne})$ ist das neue (v'́os) Element und neodym $(\mathrm{No})$ das neue Dym als anderer Bestandtheil des Didyms, von dem schon vorher die Rede gewesen ist. Xenon $(\mathrm{X})$ ist im hochsiedenden Antheil des flüssigen Argon entdeckt und, 
508 Diergart: Etymologische Untersuchungen u.s. w.

um auf sein seltenes Vorkommen überhaupt und seine Ent-

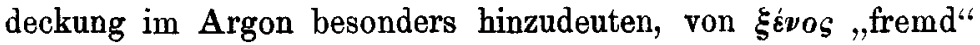
(Fremdling, Gast) so benannt worden. - Alles dies sind zwar alte, aber nach ihrem Sinne neue Wörter. Alte Wörter, die schon früher wesentlich dasselbe bedeutet haben, finden sich in den Namen unserer Elemente gleichfalls.

\section{Benennungen nach Stoffen, in denen die Elemente vorkommen.}

Die metallischen Eigenschaften des elementaren arsenum (As) sind nach Albertus Magnus schon im 13. Jahrhundert, vielleicht auch noch früher, bekannt gewesen, denn er schreibt in seiner Schrift "de alchymia": ,arsenicum fit metallinum fundendo cum duabus partibus saponis et una arsenici". "Arsericum" ist offenbar übernommen aus dem Griechischen.

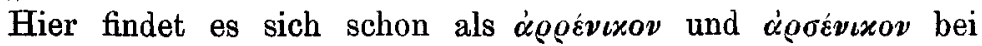
Aristoteles' Schüler Theophrast und bei Dioskorides, der darunter das Auripigment $\left(\mathrm{As}_{2} \mathrm{~S}_{3}\right)$ verstanden $\mathrm{zu}$ haben

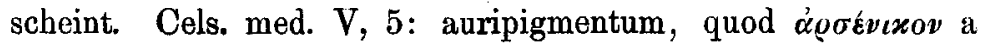
Graecis nominatur. Gelbe Farbe ćgoźvıxov = Operment s. John (Malerei der Alten), S. 128. Es ist sehr unwahrscheinlich, dass dem Mineral jener Name sinnbildlich beigelegt worden ist wegen der stark giftigen Wirkung seines Röst-

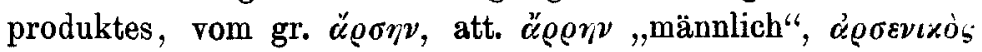
, von männlicher Art". Denn wenn auch in der philosophischen Mystik das Wirksame im Gegensatz zum Leidenden zuweilen das Männliche ( $\tau$ ò $\alpha \varrho \varrho \varepsilon v$ ) genannt wird, so liegt doch kein Grund vor, den Namen bei den Mystikern zu suchen. Die chemische Mystik ist fast 1000 Jahre später als Theophrast. Vor allem aber heisst der Stoff nicht ă $\varrho \rho v$, sondern áọévıxov, d. h. etwas, was Bezug hat auf das Männliche. Es ist also wohl ein passendes Hauptwort als Träger zu suchen. Die mich persönlich am meisten befriedigende Ableitung, die zugleich allen Ansprüchen der Etymologie und des Sinnes entspricht, verdanke ich einer gütigen Privatmittheilung des Herrn Geh. Reg.-Raths H. Diels, ord. Prof. a. d. Univ. und ständiger Secretär der Kgl. Akad. d. Wiss. zu Berlin. Er ergänzt $\chi \rho \omega \bar{\mu} \mu \alpha$ und bezeichnet das Operment als Männerfarbe. Die ältere griechische Malerei arbeitet nur mit ganz wenig 
Diergart: Etymologische Untersuchungen u. s. w. 509

Farben, darunter mit Gelb, das bei der Darstellung des Menschen so verwendet wird, dass es die sonnengebräunten Männer im Gegensatz zu den weiss (Bleiweiss) gemalten Weibern gelb tönt. Denn die in der Gynaikonitis eingesperrten Frauen stechen, wie noch heute im Orient, durch ihre Hautfarbe lebhaft von den Männern ab.

Von Kobell ${ }^{1}$ ) bringt Arsen mit einem arabischen arsa naki zusammen, das ,ein tief in den Körper eindringendes Unglücksgift" sein soll, in Wirklichkeit aber in der arabischen Sprache nicht vorkommt. Hier dürfte eine Warnung am Platze sein, arabische Worte zur Vergleichung mit offenbar arischen Ausdrücken herbeizuziehen. Die semitische Rede und die arische Weise (früher indogermanische genannt) sind durchweg verschieden in ihren Stammformen. "Arsenik" kommt schon bei Plinius dem Aelteren (23-79 n. Chr.), also im ersten christlichen Jahrhundert in seiner berühmten, aus etwa 2000 griechischen Werken geschöpften „historia naturalis“, XXXIII, 18 vor, also etwa sieben Jahrhunderte, bevor die arabische Litteratur in der Kulturgeschichte eine Rolle spielt. Zudem kennen die Araber sowohl als die Perser das Arsen als zirnuk $(\mathrm{z}=$ weiches $\mathrm{s})$ und pers. zirne. Diese stammen offenbar aus dem Griechischen, $r$ und $s$ umgestellt. Es giebt freilich im Semitischen den Stamm razan „schwer gewichtig sein", auch giebt es rasan, Mehrzahl ersan "Schiffstau", aber quid hoc sibi vult? Wollte man in kühnem Schwunge vom "Schwer sein" Arsen ableiten, müsste man doch die historischen Mittelglieder zwischen der Antike und dem Mittelalter suchen. Leider ist das Buch $\pi \varepsilon \rho i \lambda i \vartheta \omega v$ des Aristoteles verloren gegangen, denn hier würden offenbar die Grundzüge der antiken Geologie und Mineralogie aufzudecken sein.

Eher arabischen Ursprunges ist der Name des Elementes Antimon. Antimonium kommt schon bei Constantinus Afrikanus (um 1100) zuerst vor. Daher ist seine Ableitung von "antimonachum - gegen den Mönch" in Bezug auf die bekannte Erzählung bei Basilius Valentinus (15. Jahrh.); endlich mal ad inferos zu schaffen. Zudem sagt er selbst in seinem "Triumphwagen des Antimonii“" nach einer Be-

1) Gesch. d. Mineralogie, München 1864, S. 536. 
sprechung des stibium $\left(\mathrm{Sb}_{2} \mathrm{~S}_{3}\right)$; ,in der lateinischen Sprache hat man es bis auf den heutigen schwebenden Tag Antimonium geheissen." Ob das Antimon aus dem arab. das (al) ,it mid" herzuleiten ist, erscheint der neuesten Forschung noch fraglich. Jedenfalls ist aber Zusammenhang vorhanden; denn nach $\mathrm{Jakob}^{1}$ ) bezeichnet itmid die Augenschminke nach ihrer Substanz und kohl die Augenschminke als Schönheitsmittel. it mid soll nach Fränkel ${ }^{2}$ ) auf das ägypt. mștm zurückgehen. Das gr. $\sigma \tau i \mu \mu \iota, \sigma \tau i \beta \iota$, (Schwefelantimon) führt er gleichfalls auf mṣtm zurück und leugnet mithin die bisher vertretene Ent-

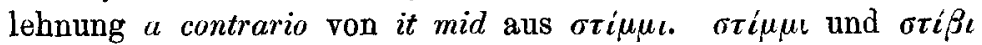
ist ins Lat. übergegangen als stimmi und stibi. Plin. 33, 101. „Stimi appellant, alii stibi, ..." Hieraus ist stibium geworden, von dem das Sigel Sb herrührt. Das Zeitwort $\sigma \tau \iota \beta i \zeta \varepsilon \imath \nu$ „mit Spiessglanz schminken" bei Ezechiel in der SeptuagintaUebersetzung des alten Testaments ist aus $\sigma \tau i \mu \mu \iota$ gebildet

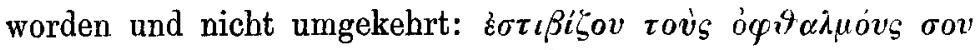
(du schminktest deine Augen mit Spiessglanz).

Ebenso alt ist das gr. $\mu o \lambda v ́ \beta \delta \alpha \iota v \alpha$, das sich in unserem Molybdän (Mo) erhalten hat. Was Dioskorides unter $\mu$ óiv $\beta$ $\delta \alpha \iota \nu c$ versteht, scheint wesentlich Bleiglätte gewesen zu sein, obwohl es eigentlich „Bleikugel" heisst. Allgemein ist augenscheinlich sowohl im Griechischen wie bei Plinius unter moiybdaena ein bleihaltiger oder bleiartiger Körper verstanden worden, der ebenso abfärbt wie das $\mu o ́ \lambda v \beta o_{s}$, dessen $A b$ leitung oben erwähnt ist. Später hat man darunter nur die Mineralien verstanden, die ebenso abfärben wie die Bleierze. Schliesslich hat sich die Bezeichnung nur auf den Molybdänglanz und den Graphit übertragen, die lange Zeit miteinander verwechselt und für gleichartig gehalten worden sind. Nach Feststellung der Verschiedenheit der beiden Mineralien ist in dem ersten das neue Element entdeckt worden, dem der alte Name Molybdän zu Theil geworden ist.

Vielleicht ähnlich liegen die etymologischen Verhältnisse des borum $(\mathbf{B} \phi)$. Denn aus $\mathrm{Geber}^{3}$, bei dem sich das Wort

1) „Altarab. Beduinenleben", Berlin 1897.

2) „Die aramäischen Fremdwörter ion Arabischen", Leiden 1886.

$\left.{ }^{3}\right)$ Eigentlich soll er Abu-Mussa-Dschafar-al-Sofi geheissen haben; 
Diergart: Etymologische Untersuchungen u.s. w. 511

borax oder baurach wahrscheinlich zuerst findet, geht nicht deutlich hervor, ob dieser "Borax" mit unserem heutigen "borum" chemisch überhaupt etwas gemein hat, um so mehr als sein ,Landsmann Avicenna unter baurach unser fixes Alkali versteht. ${ }^{1}$ ) Die Annahme ist um so berechtigter, als Geber nach Kopp die Anwendung des baurach zu Glasflüssen erwähnt. ${ }^{2}$ ) Im Hebräischen ist unter dem Wortlaute borit wahrscheinlich Pottasche gemeint. "borax" hängt vielleicht mit dem arab. baraka "blitzen" zusammen. Der Blitz heisst darnach bark, das vom leuchtenden Stern vielfach gebraucht wird. Wie dem auch sei, jedenfalls hat das Bor seinen Namen vom Borax, ebenso wie vom Zirkon = Edelstein der Name des Elementes zirconium $(\mathrm{Zr})$ herrührt. Dieses Edelsteines erwähnt schon Plinius $(37,153)$ unter der Benennung circos, das, was $W$ eise bestätigt, dasselbe ist wie das gr. xi@xos. Plinius H. N. XXXVII, 153: "- ceritis cerae similis est, circos accipitri, corsoides canitiae capitis, .... gignitur in India (Ceylon) et Syene -." Ki oxos oder, was dasselbe bedeutet, $x \dot{x} \lambda_{o s}$ (W. $x v \lambda, x v \rho$, Skr. ca-kr-a = rota, orbis) ist „Ring“. Da nun der Circos-Krystall (Prismen mit pyramidalen Endflächen) nur im polarisirten Lichte ringförmige, mit blossem Auge nicht sichtbare Gebilde um die senkrechte Axe aufweist, so ist es ausgeschlossen, dass von dieser Eigenschaft der Name herrührt. (Narrata refero) ${ }^{3}$ ) s. Nachtrag 3.

Das lat. carbo, von dem das Element carbonium (C) seinen Namen hat, bringt Hintner mit cremare in Zusammenhang (W. kar-, kal-, „brennen, flammen", Skr. grā- [aus çar-] kochen, kal-ma-li "das Flammen". Im Deutschen heisst das Element „Kohlenstoff“. Das zu Grunde liegende „Kohle“ (ahd. kolo)

aus der Abkürzung dieses Namens Dschafar soll im Munde der Abendländer Geber geworden sein, er hat im 8. Jahrhundert gelebt.

1) S. Kopp, Geschichte d. Chem. Bd. 3, S. 339. Leider ist es mir nicht gelungen, die Stellen bei Geber und Avicenna zu finden.

2) S. Kopp, Bd, 3, S. 339. Ferner s. Nachtrag 2.

${ }^{3}$ ) von Kobell (S. 478) bringt Zirkon mit dem franz. jargon zusammen, was bei den älteren Schmuckhändlern einen diamantähnlichen Stein bedeutete. Die Annahme ist wenig wahrscheinlich.

Georges (Lat.-Dtsch. Wb., Leipzig 1869, 7. Aufl.) sagt, circos bedeute "Habicht" und ,einen Edelstein von der Farbe des Habichtes" Der Zirkon ist aber sehr verschieden gefärbt. S. Nachtrag 3. 


\section{Diergart: Etymologische Untersuchungen u. s. w.}

vergleicht man (Kluge) als urverwandt mit Skr. jval ,brennen, glühen". In näheren Zusammenhang stellt Grimm das nord. Wort, schw. kylla, kölla ,einheizen, Feuer im Ofen machen", auf das schon Adelung ${ }^{1}$ ) verwiesen hat. Dazu kol -- feuerfasz bei Riez ${ }^{2}$ ) 343 und koljarn, Feuerstahl, in der Form ganz zu ,,kol, kohle" übertretend. ${ }^{3}$ )

Bestimmteres ist über die Ableitung von Fluor (F) anzugeben. Diesen Namen verdankt es der Eigenschaft des Minerals, in dem es sich findet, des Flussspats, das Schmelzen der Erze zu beschleunigen, sie rascher fliessend zu machen (fuere "fliessen"). Der erste, der sich über den Flussspat deutlicher ausspricht, scheint Agricola (im 16. Jahrh.) zu sein. Er sagt in seiner Schrift „Bermannus sive de re metallica dialogus", nachdem er eingehend über eine gewisse Steinart (Flussspat) gesprochen hat: - - - reddunt enim materiam in igne non paulo fluidiorem", und in Libavius' ,alchymia" wird es häufig als fluor mineralis bezeichnet, woher dann der Name des darin enthaltenen neuen Elementes entnommen ist. - Nicht bis in die graue Vorzeit zurück geht die Kenntniss der Baryterde, nach der das baryum (Ba) benannt worden ist. Das erste Baryum-Mineral, auf welches man aufmerksam geworden ist, war der oben erwähnte Litheosphorus, der im Deutschen den Namen Schwerspat wegen seiner Schwere bekam. Nach der Entdeckung der darin enthaltenen neuen Erde wurde diese terra ponderosa (Schwererde) genannt, in Frankreich nach $\mathrm{K}$ opp (Bd. 4, S. 44) angeblich barote vom gr. $\beta \alpha \varrho v_{\zeta}$ schwer. $\left.{ }^{4}\right)$

Die französische Benennung ist in "Baryt" verändert in die antiphlogistische Bezeichnungsweise übergegangen.

Bedeutend älter ist die Wurzel, die dem Wort natrium (Na) zu Grunde liegt. Das am längsten bekannte Natriumsalz ist die Soda. Ihrer wird sehr wahrscheinlich schon im alten

1) Chr. Adelung, ,magazin für die deutsche sprache“, Leipzig 1783.

2) J. E. Rietz, ,ordbock öfver svenska allmoge spräket", Lund 1862.

3) Die ,Пlias post Homerum" von Freudenberger, carbo mit ahd. raban (Rabe) zusammenzubringen bei Zugrundelegung einer idg. Grundform ,krabb" schwarz, ist ungereimt.

4) Der Wechsel von $v$ in 0 ist verdächtig, wahrscheinlich liegt ein Druckfehler statt baryte vor; Kopp's Quelle zu barote habe ich nicht entdecken können. 
Diergart: Etymologische Untersuchungen u. s.w. 513

Testament als than. Sprüche 25, 20: „wer ein Kleid auszieht in der Kälte, so ist das wie Essig auf neter“. Jeremias 2, 22: „neter dient vermischt mit Oel als Seife; wenn man Wasser darauf giesst, schäumt es," (Verseifung); daher von natar = aufspringen.")

Nach Curtius und Benfey ${ }^{2}$ ) ist dem hebr. Wort das gr. vitoov entlehnt, das man sehr lange für gleichbedeutend mit Salpeter gehalten hat. Jedoch hat die semasiologische Untersuchung, die stets jeder etymologischen vorauszugehen hat, bereits ergeben, dass das vitoov bei Dioskorides und das nitrum bei Plinius nicht als salpeter-, sondern als kohlensaures Salz zu verstehen ist, sehr wahrscheinlich sogar als Soda. Denn bei der Angabe seiner Darstellung wird die Gewinnung aus (natron)-Seen in erster Linie erwähnt. S. Nachtrag 4. Neben vielen anderen Stellen ist folgende bei Plinius die am meisten kennzeichnende, wo er bei einer Beschreibung des nitrum sagt: ,igni non exsilit nitrum". Da ihm nun das ,igni exsilere" in Bezug auf das Kochsalz bekannt gewesen ist, so hätte er sicherlich dasjenige des nitrum, wenn es Salpeter gewesen wäre, erwähnt. Biringuccio (im 16. Jahrh.) unterscheidet ausdrücklich das nitrum vom Salpeter, den man des Unterschiedes wegen sal nitri genannt habe. ${ }^{3}$ )

Etwas früher ist durch die Araber der Ausdruck natrun, natrum, nutron statt nitrum in Europa gebräuchlich geworden, das wahrscheinlich mit vitoov in Zusammenhang steht. Unter natron hat man von nun an das mineralische (kohlensaure) Laugensalz verstanden, während der Salpeter kurzweg nitrum genannt worden ist (s. unter N). Und nach dem Natron ist sein metallischer Bestandtheil zuerst natronium, später abgekürzt natrium benannt worden. Mit der Geschichte des Namens natrium ist diejenige des Namens kalium (K) aufs engste verknüpft, zumal die Salze beider, besonders die kohlensauren, bis über die Mitte des vorigen Jahrhunderts für gleichartig gehalten, wenigstens nicht streng gesondert worden sind. Der Ausdruck kali oder mit dem Artikel al-kali findet

1) S. Gesenius, „Hebräisches Wörterbuch“.

2) Griech. Wurzellex., Berlin 1872.

s) Salpeter hat einen salzigen Geschmack und Soda nicht. 
sich zuerst bei arabischen Schriftstellern und ist in seiner Bedeutung wahrscheinlich gleich vitgov. kali oder al-kali sind vermuthlich bei den Arabern die Pflanzen ${ }^{1}$ ) benannt worden, durch deren Verbrennen sie den erwähnten Stoff erhalten haben, und dann auch dieser selbst, weil sie unter dem Wortlaute qiljin, qaljan "Asche" verstanden haben, das natürlich mit dem Zeitwort qalaj ,rösten, im Tiegel kochen“" in Beziehung steht. kali, al-kali und natrum bezeichnen noch im 16. Jahrh. dasselbe. Von da an ist die Chemie fast nur von Deutschen, Franzosen und Engländern betrieben worden, und es sind daher zur Herstellung von al-kali gewöhnlich nur Binnenlandpflanzen (also mit $\mathrm{K}$ ) verwendet worden.

Was man nun al-kali genannt hat, ist also gewöhnlich Kali (Pottasche und Weinsteinsalz) gewesen. Als man nun in der Kochsalzbasis ein eigenthümliches Alkali entdeckt und sich die Verschiedenheit von kali und natrum herausgestellt hatte, hat man dieses fixes mineralisches und jenes fixes vegetabilisches Alkali genannt. Da diese Bezeichnung sich nicht als vortheilhaft erwies, hat man die Namen potassinum (Pottasche) und natrum eingeführt, die jedoch meist auf die kohlensauren Alkalien bezogen worden sind. Die Bezeichnungen potasse und soude (Soda) sind dann in Bezug auf die ätzenden Alkalien eingeführt worden. Diese Abkürzung hat man im Deutschen erreicht, indem man Kali und Natron in den noch jetzt ihnen beigelegten Bedeutungen gebrauchte, nämlich zur Bezeichnung der ätzenden Alkalien. Der metallische Bestandtheil des Kali hat dann den Namen Kalium (K) bekommen. ${ }^{2}$ )

Ein anderes altes Wort ist das ,alumen" = Alaun der Römer, nach dem das darin befindliche „Aluminium" (Al) benannt worden ist. Plinius unterscheidet sogar mehrere Arten des alumen, das wahrscheinlich angewendet worden ist, um die Wolle zum Färben vorzurichten und die Farben an die Stoffe zu binden. Plinius sagt XXX, 190: ,ad reliquos usus vitae in coriis lanisque perficiendis quanti sit momenti (alumen)

1) Gattung Salikornia s. Freitag, ,Arabisches Wörterbuch".

2) Dies nach Freudenberger aufs hebr. qal (nicht kal) "leicht" zurückzuführen wegen des leichten specifischen Gewichtes, ist ausgeschlossen. 
Diergart: Etymologische Untersuchungen u.s.w. 515

significatum est." Nach Isidorus im 7. Jahrh. soll alumen von lumen sogar seinen Namen baben, denn er sagt XVI, 2: ,alumen vocatur a lumine, quod lumen coloribus praestat tingendis." Er nennt es auch salsugo terrae ebenso wie Plinius H. N. XXXV, 15 med. sect. 52: „nec minor est aluminis opera, quod intelligitur salsugo terrae." Die hierauf fussende Ableitung rom griech. $\ddot{\alpha} \lambda u \eta$ (alles was salzig ist), $\ddot{\alpha} \lambda_{s}$ wie überhaupt jede Herbeiziehung des Griechischen ist wohl ausgeschlossen. Dasselbe bezieht sich auf die Erklärung von Vossius (Etymol. in Aluta), der es vom gr. "̌c $\lambda \varepsilon \varepsilon \mu \mu c$ (Fett, Oel, womit man salbt) i. e. unguentum herleitet. Dieses würde mit á̉ $\lambda \varepsilon i \varphi \omega$, ungo salben, Skr. W. anj - salben, schmücken in Beziehung zu bringen sein. Alle diese Erklärungen lassen sehr viel zu wünschen übrig, namentlich diejenige von Isidorus, die wahrscheinlich seine eigene Erfindung und ganz unbegründet ist. Deshalb ist es wohl richtiger, sich Weise's Ansicht anzuschliessen, für den alumen ein im Latein allein vorkommender und unaufgeklärter Name ist. Vanicek äussert sich nicht darüber. S. Nachtrag 5 .

Eine ebenfalls dem Latein eigenthümliche Benennung ist die des silex - was nach Weise keinem Zweifel unterliegt dessen metalloïdischer Bestandtheil unser Element silicium (Si) ist. Blümner ${ }^{1}$ ) will den silex unmittelbar der Härte wegen so benannt wissen, wie denn die Römer darunter auch jeden härteren Stein (gabinische und albanische Steine) verstanden haben, im Gegensatz zum weichen lapis Tiburtinus, Sinter, weisser Kalkstein, der gewöhnlich mit calx übersetzt ist. ${ }^{2}$ ) Von ihm hat das Element Calcium (Ca) als metallischer Bestandtheil seinen Namen. Nach Curtius, Weise und Vanicek, um der gegentheiligen Ansicht Freudenberger's entgegenzutreten, liegt dem cal-c-s wahrscheinlich das gr. $\chi^{\alpha} \alpha^{\prime}-t-x-s$ $(\chi \alpha \dot{\lambda}, \xi)$ zu Grunde, wie die Bereitung und Verwendung des Mörtels calx den Römern erst mit der Steinbaukunst durch die Griechen bekannt geworden sein dürfte. $\left.{ }^{3}\right)$ Nach Curtius gehören diese beiden Wörter mit Skr. çarkarâ „Kiesel, Scherbel“"

1) "Terminologie und Technologie der Gewerbe und Künste bei Griechen und Kömern", Leipzig 1887.

2) Weiteres iber silex siehe Nachtrag 6 .

3) Siehe Nachtrag 6, Anmerkung. 
zusammen, dem das Eigenschaftswort Skr. khara „,hart, rauh“ zu Grunde liegt, so dass $\chi \chi^{\prime} \lambda \iota \xi$ und calx eigentlich das „Harte“ bedeuten. S. Nachtrag 7.

Aus der Zeit des klassischen Alterthums führt uns die Etymologie des Namens Wolfram (W) in das germanische Mittelalter hinüber. Dem Worte in dieser Bedeutung begegnen wir zuerst etwa im 15. Jahrh. Es ist in den Zinnhütten der damaligen Zeit wahrscheinlich sinnbildlich einer Beimengung des Kassiterits (Zinnerz) gegeben worden, durch die der Zinngehalt beim Zinnschmelzen durch Schlackenbildung vermindert wird.") "Wolfrig" heisst soviel wie ,fressend". Die Endung ram ist die Verkürzung von raban (Rabe). Die Zusammenstellung von Wolf und Rabe ist mythologisch. ${ }^{2}$ )

Ausser der Benennung nach Eigenschaften sind die Mineralien und sonstigen Stoffe zuweilen auch nach Forschern benannt worden. Zu ihnen gehören aus neuerer Zeit der Samarskit nach dem russischen Bergbeamten von Samarski ${ }^{3}$ ) und der Holmesit nach Dr. Holmest), von denen dann die Namen der Elemente samarium ( $\mathbf{S} a$ ) und holmium (Ho) entnommen worden sind. Etymologisch ähnlich zu erklären ist vielleicht die cadmia fornacum (Zinkofenbruch), nach der das Element cadmium benannt worden ist. Der Name xuducice findet sich schon bei Dioskorides und als cadmia bei Plinius. Er ist vielleicht mit Kadmos, dem Sohn des Agenor und der Telephassa in Beziehung zu bringen, der die $K a ́ \delta \mu o v$ $\left.\tau \tau^{\prime} \chi v \eta^{5}\right)$ aus Phönizien nach Griechenland gebracht haben soll $\left.{ }^{6}\right)$. K $\boldsymbol{c} \delta \mu \varepsilon \tilde{i} \alpha$ scil. $\gamma \tilde{\eta}$ mit ,thebanischer Erde" als örtliche Benennung aufzufassen nach Berendes, ist nicht ganz unmöglich, lässt sich aber nicht beweisen. Denn als Heimath des natürlichen Galmei

1) von Kobell S. 665 und Kenngott, „Handwörterbuch der Mineralogie, Geologie, Paläontologie" in der Encyklopädie der Naturwissenschaften, 2. Abth., 1. Theil, Bd. 1. Breslau 1882.

2) Dem Siegesgott werden zwei Wölfe (Geri und Freki) und zwei Raben (hugin und munin) beigelegt; schon dem Apollo sind beide Thiere heilig gewesen.

3) von Kobell, S. 549. 4) Das. S. 498.

$\left.{ }^{5}\right)$ Them. or. 4, S. 60, Gewinnung und Bearbeitung der Erze.

6) Her. 5, 58; Plin. 7, 56, Themisonis fragmenta in Müller, hist. Graee. Vol. IV. 
Diergart: Etymologische Untersuchungen u.s.w. 517

giebt Strabo (z. Z. Chr. Geb.) vornehmlich Cypern an. Er

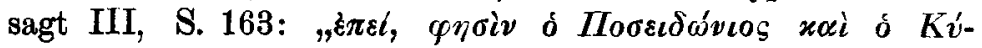

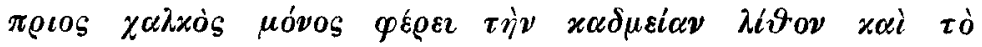

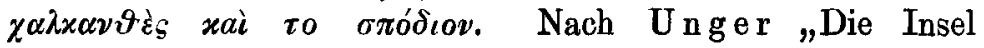
Cypern", S. 18, findet sich dort heute noch Galmei. Plinius nennt Kampanien seine Fundstätte. Ausserdem versteht Dioskorides $V, 84$ unter $\varkappa \alpha \delta \mu \varepsilon i \alpha$, was $\mathrm{Blümner}$ bestätigt, nur das künstliche Zinkoxyd (Zinkofenbruch) und nicht die natürliche cadmia, von der allerdings sein Zeitgenosse Plinius spricht: "aes fit e lapide aeroso quem vocant cadmiam." Sein Ansehen aber in Bezug auf einzelne Thatsachen ist bekanntlich sehr zweifelhaft, weil er seine Vorgänger theils schlecht verstanden und die Thatsachen theils aus Theophrast und Dioskorides vielfach ohne eigene Einsicht abgeschrieben hat.

Aehnliche Ansichten herrschen über die magnesia (nigra) der Alten, eine Benennung, nach der das Element magnesium $(\mathrm{Mg})$ und wahrscheinlich auch das Element manganium $(\mathrm{Mn})$ benannt worden ist.

Dioskorides and Plinius verstehen unter dem $\mu \alpha \dot{\alpha} \gamma \eta \eta_{s}$

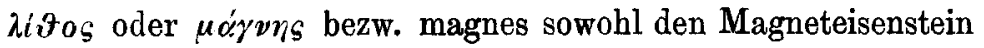
wie den Braunstein. Aus ihren Beschreibungen geht einerseits mit Sicherheit hervor, dass es sich um das Magneteisen handelt. Dioskorides V, 147, Plinius XXXIV, $147 \mathrm{ff}$., XXXVI, $126 \mathrm{ff}$.

Die Ausdrucksweise des Plinius, der magnes werde zur Bereitung farblosen Glases benutzt, weil er aus dem Glase die "verunreinigende" Feuchtigkeit herausziehe, lässt andererseits seine Uebereinstimmung mit Braunstein als sicher annehmen. Wie die $\varkappa \alpha \delta \mu \varepsilon i \alpha$ nach Kadmos, so soll, wie Plinius erzählt, der $\mu c^{\prime} \gamma \nu \eta s$ nach dem Hirten Magnes benannt worden sein, der diesen Stein auf dem Berge Ida entdeckt habe, dadurch dass die Eisenspitze seines Stockes und die Nägel seiner Schuhsohlen plötzlich am Boden festgehalten worden seien. Vergl. Plinius H. N. XXXVI, 16, sect. 25: „magnes (scil. lapis) adpellatus est ab inventore ut auctor est Nicander, in

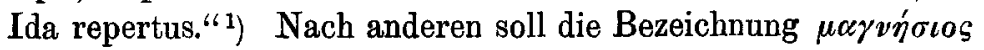
$\lambda i \vartheta$ os eine örtliche sein, was eher möglich ist als die örtliche Benennung der $x \alpha \delta u \varepsilon i \alpha$. Denn als Fundstätten des magnes

1) Siehe Nachtrag 8. 
werden genannt Aethiopien, Lydien u. a., s. Plinius. Da es nun in Lydien am Berge Sipylus eine Stadt Magnesia gegeben hat, ist eine örtliche Benennung wohl möglich. Plinius unterscheidet einen männlichen und einen weiblichen magnes. Jener ist wahrscheinlich der eigentliche Magnet, dieser der Braunstein gewesen, von dem er sagt: „magnes qui niger est et feminei sexus, ideoque sine viribus (Eisen anzuziehen) - - ." Auf diesen Unterschied sexu ist vielleicht die mittelalterliche Bezeichnung magnesius tapis für den Magneten und magnesia (nigra) für den Braunstein zurückzuführen. Bis ins 18. Jahrh. sind die beiden Mineralien für gleichartig gehalten worden, bis in der magnesia (nigra) ein neues Metall gefunden worden ist, dem man den Namen magnesium gegeben hat. - Kurz vorher war von Italien aus ein heilkräftiges Geheimmittel von unbekannter Zusammensetzung unter dem Namen „magnesia alba" in den Handel gekommen. Weshalb der alte Name auf diesen unbekannten Körper übertragen worden ist, hat selbst Kopp nicht ausfindig machen können. Vermuthlich ist es nur des Gegensatzes in der Farbe wegen geschehen, weil die blendend weisse magnesia alba vor der dunklen magnesia nigra sehr hervorsticht. Nach Kopp ist das Metall magnesium in Frankreich, England und Italien "manganes" genannt worden, da:dort die Aehnlichkeit der Bezeichnungen für schwarze und weisse magnesia jener den Namen manganesium zugeführt hatte. Dass das. Wort manganesium nur das langgezogene magnesium ist, erscheint wahrscheinlicher als seine Ableitung vom gr. $\mu c^{\prime} \gamma \gamma \alpha \nu o v$,Täuschungsmittel“, die Berendes giebt. Denn der Name "mangan" findet sich schon im 16. Jahrh., während erst Mitte des 18. Jahrh. entdeckt worden ist, dass der Braunstein kein Eisenerz ist. Zudem ist die wahrscheinlich erste Abweichung vom Ausdruck magnesia, die sich bei Camillus Leonardus ${ }^{1}$ ), einem ital. Arzt (1. Hälfte 16. Jahrh.), als "mangadesus" (vgl. $\mu \alpha \dot{\alpha} \gamma \gamma \alpha \underline{\text { pyov }}$ ) und später erst bei seinem Amtsbruder Michael Mercati²) (2. Hälfte 16. Jahrb.) als "manganensis" findet, nicht angethan, Berendes' Ableitung vom gr. $\mu \alpha \dot{\gamma} \gamma \operatorname{covov} \mathrm{zu}$ rechtfertigen.

1) in seinem "Speculum lapidum".

$\left.{ }^{2}\right)$ in seiner "Metallotheca". 
Diergart: Etymologische Untersuchungen u.s. w. 519

Im Gegentheil, da die italienische Aussprache das $\mathrm{Zu}$ sammentreffen von zwei Consonanten vermeidet, ist es glaubwürdiger, dass sich aus magnesia allmählich Bezeichnungen gebildet haben, aus denen das heutige "manganium" hervorgegangen ist. Nach der Entdeckung eines neuen Metalles in der magnesia alba ist diesem zum Unterschiede des vorher entdeckten magnesium oder manganesium der Name magnium gegeben worden. Als sich nun für jenes die abgekürzte Bezeichnung mangan eingeführt hatte, ist die Benennung magnesium auf das Metall der magnesia alba übergegangen.')

Vielleicht gleichfalls örtlich ist die lat. Bezeichnung $b e$ ryllus aufzufassen, nach dem das Element beryllium (Be) [frz. und engl. G, wie besprochen] benannt worden ist. Beryllus ${ }^{4}$ ) ist offenbar mit dem gr. $\beta$ ŕ $\varrho v \lambda \lambda o_{S}$ zusammenzubringen, das nach Weise ohne Vermittelung semitischer Völker aus dem Indischen sofort ins Griechische übergegangen ist. A. Weber ${ }^{2}$ ) stellt es mit pkr. veluriya aus skr. vandūrya zusammen. Im späteren klassischen Sanskrit ist vaidürya "Name eines Steins" (des Berylls) und wird von den Indern als von vidūra kommend gedeutet. Vidūra, wörtlich ,sehr fern", ist entweder Name eines Berges oder eines Landes. Man hat es mit „Bolor, Belur-Tagh" in Afghanistan in Verbindung gebracht. Eine andere Deutung, die jedoch den meisten Etymologen unwahrscheinlicher erscheint, hat F. Max Müller ${ }^{3}$ ) vorgeschlagen. Er fasst vaidūrya auf als aus urspr. vaidārya bezw. vaidälya entstanden. vaidälya würde bedeuten, von vidāla, d. h. von der "Katze" stammend, kurz: vardūrya sei = „Katzenauge". Eine begründete Benennung örtlicher Art ist die des bei Strontian in Argyleshire (England) gefundenen und danach benannten Strontianits, wonach das Element Strontium (Sr) seinen Namen erhalten hat. Nach Ytterby in Schweden ist die Yttererde benannt worden, und die Namen der darin enthaltenen Elemente yttrium $(\bar{Y})$, erbium $(\mathrm{Er})$, terbium $(\mathrm{Tr})$ und ytterbium $(\mathrm{Yb})$ sind Umsetzungen der Silben des Wortes Ytterby.

1) S. Kopp, „Geschichte der Chemie“".

$\left.{ }^{2}\right)$,Ind. Studien“, III, S. 178.

3) "India what can it teach us?" London 1883, S. $265 \mathrm{ff}$.

4) Siehe Nachtrag 9. 
Bezeichnungen nach dem Orte des Vorkommens.

Ebenso begründet ist die örtliche Benennung des Elementes cuprum $(\mathrm{Cu})$. Wahrscheinlich schon den alten Israeliten bekannt, ist es dort sowohl wie bei den Römern die Bezeichnuug für ,Erz" geworden, wie denn auch die Römer unter "aes" Kupfer und das kupferhaltige Messing verstanden haben. Als ersten Fundort des aes nennt Plinius Cypern: "- - in Cypro, ubi prima fuit aeris inventio - “. Deshalb ist es bald aes oyprium "Erz von Oypern", dann nur cyprium. wie bei Vitruvius und auch bei Plinius benannt worden. Die ältere lat. Form dafür ist cuprum. DieAbleitung a contrario, der Ausdruck Cypern komme von dem Namen eines Metalles kipar her, der sich auf altassyrischen Inschriften findet, ist unsicher (vergl. Beck, S. 125 ff.) Andere bringen die Pflanze kophar (arab. el-hanna) in Zusammenhang, die auf Cypern häufig vorkommt. ${ }^{1}$ ) Das lat. Wort ist nicht nur in die romanischen (cuivre), sondern auch in die germanischen Sprachen übergegangen. Es ist nach Grimm und Kluge schon frub als kupar gemeingermanisch geworden und tritt im Ahd. als kupfar, Mhd. kupfer auf. ${ }^{2}$ )

Dus Element ruthenium $(\mathbf{R u})$ ist nach Ruthenia, dem alten Namen Russlands, genannt worden. Skandinavien findet sich wieder im Namen des Elementes scandium (Sc), und von Thule, dem alten Namen für Norwegen und überhaupt das nördlichste Europa, hat das Element thulium (Tu) seine Benennung. Die Namen der Elemente germanium (Ge), gallium (Ga) und des jüngst entdeckten polonium (?) erklären sich von selbst. Weniger ist dies der Fall bei dem Namen des Elementes Wismut (Bi). Nach Kluge ist es wahrscheinlich nach der ältesten Wismutzeche St. Georgen ,in der wiesen“ bei Schneeberg benannt worden; muten (daher Wismut ohne h zu schreiben) ist ein bergmännisches Wort und hat die Bedeutung ,um das Recht nachsuchen, eine Zeche zu eröffnen." Im sächs.-böhm. Erzgebirge hat es durch sogenannte Volks-

1) S. Engel, „Kypros“, Berlin 1841.

2) Freudenberger's Ansicht, dem ,Kupfer" die Zugehörigkeit zu euprum zu nehmen und es mit dem ir. goba = "Schmied" zusammenzubringen, ist gegenüber dieser ganz natiurlichen Entwickelung unnütz. 
Diergart: Etymologische Untersuchungen u.s.w. 521 etymologie auch "wiesmat" geheissen, = "wiesenmatte", s. Georg Agricola „De re metallica“.

Nach Joh. Mathesius' „Sarepta“ (Nürnberg 1578) ist dies ein Name, der von den alten Bergleuten gebraucht wird, „da es blühet wie eine schöne wiese (wiesmatte, wismat), darauf allerlei farbige blumen stehen", in Bezug auf seine Eigenschaft, häufig in mannigfaltigen Farben anzulaufen. $\left.{ }^{1}\right)$

Bei Basilius Valentinus (um 1450) und bei Georg Agricola (De re metallica) findet es sich ins Lateinische übertragen als wismutum oder, um angeblichen Lautgesetzen gerecht zu werden, bisemutum. Ihm ist das Sigel (Bi) entnommen worden.

\section{Benennungen nach mythischen und sagenhaften Personen.}

Während es nur eine unbegründete Vermuthung war, das "Winsmat" mit der Götterlehre zusammenzubringen ${ }^{1}$ ), so handelt es sich beim Element vanadium $(\mathrm{Va})$ um die feststehende Thatsache. Denn es ist nach Vanadis, einem Beinamen der nord. Göttin der Liebe und Fruchtbarkeit Freya, von einem Schweden so benannt worden. Dem anord. Donnergott Thor zu Ehren hat das Element thorium (Th) seinen Namen erhalten. Auch der Name des Elementes titanium (Ti) ist mythologisch; denn die Elfenkönigin Titania, Gemahlin des Oberon, die ihren Namen von den griechischen Titanen führt, ist hier das medium nominans. Vielleicht ist das Element auch nach dem Planeten gleichen Namens so benannt worden, wie es um 1800, wo alle diese Elemente entdeckt worden sind, häufig geschieht. ${ }^{2}$ )

Diese Annahme ist um so wabrscheinlicher, als es des Entdeckers (Klaproth) Vorliebe gewesen ist, die von ihm gefundenen Elemente nach Planeten zu benennen, wie auch seine

1) Die Endung - mat nach Berendes auf die ägypt. Göttin ,mat" zurückzuführen, die mit einem Strahlenkranze von Pfauenfedern abgebildet wird, ist eine ganz unmögliche Ableitung.

2) Titania (3. Uranusmond) ist am 11. Jan. 1787 von Herschel entdeckt und benannt worden. Becker (Sonne und die Planeten), Leipzig 1883, S. 153. 
Benennung des Elementes uranium (U) nach dem einige Jahre vorher entdeckten Planeten Uranus beweist. Das Element cerium (Ce) hat seinen Namen rom Planeten Ceres ${ }^{1}$ ), ebenso wie das Element palladium $(\mathrm{Pd})$ nach dem Planeten Pallas benannt ist.

Interessanter als diese sind diejenigen mythologischen Benennungen, die für die in Betracht kommenden Elemente bildlich gewählt worden sind. Dies ist beim Element tantalum (Ta) der Fall. Der Entdecker hat es so benannt, um sowohl dem Gebrauche der mythologischen Benennungen zu folgen, als auch, wie er selbst sagt, „um auf die Unfähigkeit desselben eine Anspielung zu machen, mitten in einem Ueberfluss von Säure etwas davon an sich zu reissen und sich damit zu sättigen." Häufig gemeinschaftlich mit ihm findet sich das ihm in mancher Beziehung ähnliche niobium $(\mathrm{Nb})$, dessen Name an das verwandtschaftliche Verhältniss zum tantalum erinnern soll.

Sinnbildliche Benennungen.

Unabhängig von mythologischen Anspielungen und nur sinnbildlich ist das Element selenum (Se) benannt worden. Der Entdecker hat diesen Namen (nach dem gr. $\sigma \varepsilon \lambda \eta \nu^{\prime} \eta$, Mond) gewählt, um lediglich die Aehnlichkeit der Eigenschaften des Elementes anzuzeigen mit denen des tellurium, für das man es zuerst gehalten hat, dann auch um an das häufige Zusammenvorkommen beider Elemente zu erinnern. ${ }^{2}$ )

Auf einen ähnlichen Umstand sucht Berendes die Erklärung von „Kobalt“" zurückzuführen, indem er es von xó $\beta \alpha \lambda o$ s „Schmarotzer" ableitet, weil das Kobalt meist in Gesellschaft des Nickels vorkommt. Diese Auffassung ist unrichtig, denn der Ausdruck "Nickel" in dieser Bedeutung kommt zum ersten Male erst etwa 300 Jahre später als das Wort „Kobalt" in diesem Sinne vor, zu schweigen, dass man damals noch nichts von einem Metalle "Nickel" gewusst hat. Wahrscheinlich geht diese irrthümliche Ansicht auf eine Stelle in Agricola's „de animantibus subterraneis liber. Bas. 1549 zurück, wo dieser von den Dämonen spricht und fortfährt: „sunt deinde

1) S. Kopp, 4, 69. 2) Das. 3, 338. 
Diergart: Etymologische Untersuchungen u.s.w. 523

mites, quos Germanorum alii, ut etiam Graeci, vocant cobalos, quod hominum sunt imitatores." "Cobalus" ist aber das ins Latein übertragene „Kobolt" oder "Kobalt", denn dieses Wort findet sich schon bei Basilius Valentinus, wie bei der Besprechung des Zinks erwähnt worden ist. Ferner ist es Agricola's Absicht gewesen, alle bergmännischen Ausdrücke ins Lateinische zu übersetzen, wie sich aus dem Verzeichniss ergiebt, in dem er zum besseren Verständniss seiner Schriften die bergmännischen und von ihm ins Lateinische übertragenen Ausdrücke zusammenstellt. Ausserdem ist zur Zeit, als das Wort „Kobalt, Kobolt“" zuerst gebraucht worden ist (15.Jahrh.), nichts rom Vorhandensein des Metalles Nickel (erst 1751) bekannt gewesen; sogar die Bezeichnung Kupfernickel, wo der A usdruck "Nickel" zum ersten Male mineralogisch angewendet worden ist, wird erst 1694 von Hiärne gebraucht. Kluge führt es ausserdem in seinem Verzeichniss der deutschen Wörter aus dem Griechischen nicht an. „Kobalt" hat vielmehr nichts mit dem Griechischen und Lateinischen zu thun, sondern ist echt germanischer Abstammung. Sehen wir, wie sich Grimm und Kluge darüber äussern. Das mhd. kobolt heisst „neckischer Hausgeist". Das erste Wortelement ist anord. kofe, angs. cofa, Gemach, Kammer. „Kobolt" und "Kobalt" ist ursprünglich ein Wort gewesen mit den beiden noch heutigen Bedeutungen. Man hat darunter eine Art von Berggeistern und eine Art von Mineralien verstanden, wie aus den soeben und beim Zink angeführten Stellen Agricola's und Valentinus' hervorgeht. Das Mineral hat der Bergmann nach dem gespenstischen Bergmännchen benannt, der ,das silber raube und das schlechte erz wie böses wetter und anderes unheil sende."

Nach J. Chr. Adelung ${ }^{1}$ ) hat bei den Bergleuten alles „kobalt" geheissen, was beim Schmelzen kein Metall giebt. J. G. Krünitz ${ }^{2}$ ) sagt, der Bergmann pflege alles sogar, was er nicht kennt, „kobalt" oder „,kobolt" zu nennen, und der Hüttenmann könne schlechte Erze, die oft reich aussehen, nicht ärger schimpfen, als wenn er sie ,kobolt" nennt. So ist

1) „magazin für die deutsche sprache“, Leipzig 1783.

$\left.{ }^{2}\right)$ ökonom.-technol. encyklopädie u. s. w., Berlin 1773. 
524 Diergart: Etymologische Untersuchungen u. s. w.

bald ,kobolt" bald ,kobalt" in denselben Bedeutungen gebraucht worden. Später hat man unter kobalt mehr die Erze verstanden, die das Glas blau färben, und auf ihren metallischen Grundstoff ist dann schliesslich der alte Name "Kobalt" übergegangen, der als cobaltum scil. elementum das Sigel Co giebt.

Mit der Geschichte des Wortes „Kobalt"“ ist die des Wortes „Nickel“ auf das engste verbunden. Der mhd. Ausdruck „nickel", d. h. „Nikolaus", findet sich u. a. als Gattungsname mit verschiedenem Nebensinne namentlich als Scheltund Schimpfwort gegen Personen beiderlei Geschlechts. Denn Fischart sagt in seiner, ,klag des ehstands" (1614): ,sie werden so einen höltzernen Peter nicht zum ratsherrn machen... wer wird denn einen solchen nickel lassen oben an gehen!" Der Ausdruck nickel $=$ Hure findet sich gar oft. Der nickel oder nach niccolum (Ni) auch das nickel ist gleichzeitig auf Sachen angewendet worden, wie der Ausdruck „Kupfernickel“ beweist, der wie gesagt von Hiärne 1694 zuerst erwähnt wird. Der Farbe wegen ist es zuerst für ein Kupfererz gehalten worden. Da sich jedoch daraus kein Kupfer herstellen liess, ist es zu jener Benennung nach dem ,neckenden Berggeist" gekommen. Nachdem man ein neues Metall darin entdeckt hatte, hat dasselbe den aus dem Mineral abgekürzten Namen ,Nickel" erhalten.

\section{Benennungen nach Forschern.}

Nach verdienstvollen Männern benannt sind die wenigsten and dabei diejenigen Elemente, deren Vorhandensein am aller fraglichsten ist. Am sichersten erscheint das nach dem Chemiker Gadolin benannte, im Samarskit gefundene gadolinium (Gd). Die Elemente philippium (Ph) und mosandrium (Mo) nach Philipp Plantamour bezw. Mosander ${ }^{1}$ ) sind wahrscheinlich mit dem besprochenen terbium ein und dasselbe.

1) Nach den Handwörterbüchern der Chemie von H. von Fehling, und von Liebig, Poggendorf, Wöhler, Braunschweig 1890 bezw. 1891 . 


\section{Sonstiges.}

Somit wären alle Elemente besprochen, die sich in die angeführte Eintheilung einreihen lassen, und es bleiben noch einige übrig, deren Etymologie noch am wenigsten erschöpfend klargelegt worden ist. So das „Zinn“, ahd. ,zin", anord. u. a. ,tin".

Nach Kluge ist es eine gemein germanische Benennung, der sich nichts aus den verwandten Sprachen vergleichen lässt (ir. tinne scheint entlehnt). A ugust $\mathrm{Fick}^{1}$ ) leitet zein „Metallstäbchen" von zin ab. Zwischen beiden scheint Zusammenhang zu bestehen, wohl weil das Zinn den Germanen durch die Kelten zu solchen Stäben verarbeitet zugekommen ist und tains ursprünglich nur jeder ausgedehnte, in die länge getriebener Stab gewesen ist. Fick stellt 302 zin zur W. dî glänzen: [diná] glänzend, (vgl. skr. diná, Tag), also zin das glänzende Metall. Diese Ableitung ist jedoch sehr unwahrscheinlich. Denn die Germanen haben das Metall nicht in ihrer asiatischen Heimath, sondern vermuthlich erst in Germanien durch keltische Einfuhr kennen gelernt, was schon Schade ${ }^{2}$ ) sagt. Nach Jakob Grimm (Deutsche Grammatik, Göttingen 1822) ist angls. tin, dann ahd. verschoben zu zin, aus lat. stannum entlehnt. Aber dies ist im Latein ganz vereinsamt; es liegt nahe, dass es keltischen Ursprungs ist: bret. stean; ir. stan. Heinrich Leo (Angels. Glossar, Halle 1872) legt ihnen das Sanskritwort stha "stehen" zu Grunde (s. unter stannum). Wenn man das anlautende $s$ als ursprünglich festhalten will, so könnte man eher an skr. stan, gr. $\sigma \tau \varepsilon v \omega$, stöhnen, seufzen, denken und das Zinn onomatopoëtisch nach seinem sogen. Geschrei benannt sein lassen. Diese Ansicht, die auch von Berendes vertreten wird, ist aber nicht haltbar. Denn das "Schreien" wird augenscheinlich zuerst erwähnt in Geber's „Summa perfectionis magisterii": „Jupiter (Zinn) est corpus metallicum album, non pure lividum et sonans parum." Adolphe Piktet (Les origines indoeuropéennes, bis 1882 .

1) Vergleichendes Wörterbuch der idg. Sprachen 2, 756, Göttingen 1868.

$\left.{ }^{2}\right)$ Oskar Schade, Altdeutsches Wörterbuch, 2. Aufl., Halle 1872 


\section{Diergart: Etymologische Untersuchungen u. s. w.}

Paris 1859) stellt kymr. ystean eigtl. „Ausdehnung" zu taen "ausgedehnt", bret. stean, kymr. ystean sei in seiner eigtl. Bedeutung ,dehnbares Metall"; stannum habe zur Voraussetzung eine Form stavnum oder stavenum. Wie dem auch allem sei, stannum ist - ich schliesse mich hier Schade's Ansicht an - aus einem kelt. Worte entstanden, das anlautendes st hatte oder davor einen unbetonten Vokal. „Hätte aber das germanische $\mathrm{Ohr}$ in dem keltischen Worte st vernommen, so würde die germanische Zunge diesen ihr so geläufigen Anlaut zweifellos ebenfalls wiedergegeben haben. Vergl. angls. tin, es bleibt daher nur übrig, dass den Germanen ein mit $t$ anlautendes kelt. Wort zugekommen ist, dem die in diesem Falle für die Bedeutung nicht unumgänglich nothwendige Vorsatzpartikel es, is oder ys gefehlt hat (vergl. ausgestreckt und gestreckt). Und auf diese Weise lässt sich stannum mit angls. tin ohne Zwang vereinigen" (s. Schade). Ebenso wenig erschöpfend ist die heutige Kenntniss von der Etymologie des Silbers. Ahd. silbar, älter silabar, mhd. silber, got. silubr, angls. seoluber, seolfor, engl. silver, ndl. zilver, alts. silubar. Kluge sagt darüber: „Diese urgermanische Bezeichnung (Grundform silubra) steht in vorhistorischem Zusammenhang mit der gleichbedeutenden slav. Sippe vom aslov. sirebro, lit. sidabras. Gewiss ist das vorauszusetzende silobro kein idg. Wort; vielleicht haben es die Germanen auf der Wanderung von einem nicht idg. Stamme übernommen und es den Slaven übermittelt."

Freudenberger's Vermuthung, die germ.-slav. Bezeichnung des Silbers mit dem lat. sulfur zusammenzustellen, weil diese beiden Elemente häufig in der Natur zusammen vorkommen (Silberglanz) und daher das eine dem anderen den Namen geborgt haben kann, ist zu bestreiten, wenngleich eine gewisse lautliche Verwandtschaft nicht zu leugnen ist.

Wie sulfur (S) zu erklären ist, habe ich nirgends finden können. Jedenfalls hat es schon aus dem Grunde nichts mit den alten Bezeichnungen des Silbers zu thun, weil der elementare Schwefel in Italien (Sizilien) heimisch ist und deshalb auch wohl dort seine Benennung bekommen hat, allerdings unter fremdem Einfluss. Denn sulfur ist, was Weise bestätigt, 
Diergart: Etymologische Untersuchungen u. s. w. 527

wegen des inlautenden $f$ nicht echt lateinisch, sondern trägt fremdes (nicht griechisches) Gepräge (vergl. die erwähnten germ.-slav. Bezeichnungen für Silber). S. Nachtrag 10.

\section{Nachtrag.}

1. Kupfer kann wohl als das älteste Metall angesehen werden, das den Indogermanen bekannt geworden ist. Auch Gold und Silber wird meist (Curtius, Mommsen, Pictet) für indogermanisch gehalten, wenngleich $\mathbf{B}$ ehn (Culturpflanzen und Hausthiere) die Kenntniss dieser beiden Metalle in jener Zeit leugnet. Aber das Blei ist höchstens dem europäischen Sprachkreise zuzuschreiben, jedoch nicht ohne Bedenken. Blei und Zinn werden selten in den Veden genannt. (Zimmer). Das Eisen ist in Griechenland erst im Zeitalter Homer's (1000 v. Chr.) zur Anwendung gekommen, man wird es daher in Italien erst verhältnissmässig spät hergestellt haben, zumal es den Bewohnern der italischen Pfahldörfer völlig fremd gewesen ist. Aus der Verschiedenheit der Wurzeln und Stämme der übrigen Metallnamen geht hervor, dass diese erst entstanden sind, nachdem die Trennung der einzelnen Volksstämme stattgefunden hat.

2. Der Ausdruck Borax ist trotz seines italischen Aussehens wahrscheinlich arabisches Lehnwort. Nach W eise S. 156 ist der Stoff hauptsächlich im heutigen Toskana gefunden worden.

3. Glatzel leitet in Samuel Shilling's Mineralreich 1879 Zirkon vom indischen cerkon ab. Ein indisches Wort cerkon (soll wohl heissen neuindisches) giebt es nicht. Höchstwahrscheinlich ist jedoch damit das neupersische zergon gemeint, welches wörtlich "goldfarbig" heisst. Im Avesta wird hairigaona - aus dem nämlich zergon hervorgegangen ist "goldfarbig" noch als Schmückwort des Sonnengottes gebraucht. Die Entlehnung aus dem Neupersischen ist jedoch zu bestreiten, vielmehr wird zergon wie Zirkon von circos entlehnt sein. - Aus der angeführten Stelle des Plinius 37, 153 geht hervor, dass er sowohl beim ceritis - wie circos - Edelstein wohl an eine Benennung nach der Farbe denkt. Da das Wort

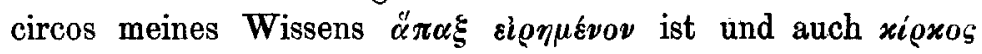


528 Diergart: Etymologische Untersuchungen u.s. w.

nicht in der Bedeutung eines Edelsteines belegt ist, so sind wir lediglich auf Vermuthungen angewiesen. xioxos bezeichnet von Haus aus den Kreis (circulus) und ist dann, aber schon bei Homer, auf den "kreisenden" Falken oder Habicht übertragen worden. $\mathrm{Ob}$ aber die kreisenden Falken alle rothbraun aussehen, möchte ich bezweifeln; ob diejenige Gattung, nach der der Edelstein benannt worden ist, diese Farbe hatte, wissen wir ebenso wenig. Der in Indien verkommende $Z_{\text {wergbussard, }}$ sowie der Rauhfuss-Bussard lassen allerdings in ihrem sehr abwechselnden Gefieder die Farben rothgrau, braun, rothbraun, schwarzbraun erkennen. Möglicherweise ist auch an die Farbe der Augen zu denken, vergl. Katzenauge u. a. Wenn also neuere Gelehrte den rothbraunen und bernsteinfarbenen Edelstein mit circos benannt haben, so beruht das sicher nur auf einer Vermuthung, deren Richtigkeit nicht bewiesen werden kann. Quantum est, quod nescimus!

Wie unsicher solche Zusammenstellungen oft sind, beweisen zahlreiche botanische und mineralogische Ausdrücke. Es genügt, die Namen des Hyazinths und der Hyazinthe in dieser Beziehung etwas genauer verfolgen, um zu der Ueberzeugung zu kommen, dass sich die modernen Namen oft gar nicht mit den antiken decken. Dazu kommt noch, dass die Farbenangaben zu allen Zeiten ungenau gewesen sind. $\chi \lambda \omega \rho o ̀ s ~ h e i s s t$ grün und gelb, caeruleus (dunkelfarbig, schwarzblau) verwandt mit dem besprochenen caesius, wird von Vergil und Ennius als poetisches Schmückwort des Himmels und des Meeres gebraucht (caerula caeli, caerula maris), Properz hat die (dunkelgrüne) Gurke caeruleus genannt. $\mu \dot{\varepsilon} \lambda \alpha \varsigma$ heisst der Wein bei Homer, und unser gelber Wein heisst Weisswein. Sed iam satis! Da Plinius der einzige ist, der den Namen circos angiebt, so ist es wohl gerathen, ihm zu glauben, zumal seine daneben stehenden Erklärungen von ceritis und corsoides richtig sind.

4. nitrum findet sich erst bei Caelius, ist jedoch weit früher bekannt gewesen als "Reinigungsmittel“. Cael. bei Cic. ad. fam. 8. 14. 4. "censuram lomentum aut nitrum esse".

5. alumen ist nach Weise $\mathbf{S}$. 156 besonders auf den liparischen Inseln gewonnen worden. 
Diergart: Etymologische Untersuchungen u. s. w. 529

6. Als die römischen Lehm- und Holzhütten durch steinerne Hüuser ersetzt wurden ${ }^{1}$ ), hat man die Arten des Baumaterials, d. h. die neuen Steingattungen nach Städten in der Nähe der Hauptsteinbrüche oder sonst nach auffälligen Eigenschaften benannt. Die Campagna hat vor Allem den silex (zu Strassenbauten), lapis Albanus (Alba) und lapis Gabinus (Gabii) (zu Wasserbauten) geliefert. Einen an silex erinnernden Ort giebt es nicht, deshalb hält Blümner's Ansicht, dass der silex wegen seiner Härte von den Römern so benannt worden sei, völlig Stand. silex bedeutet, wenn es nicht allgemein als Stein schlechtweg gebraucht wird, stets einen harten Stein und so auch übertragen wie unser Kieselstein. Ueber die Etymologie des silex weiss ich nichts zu sagen.

7. Der Widerspruch zwischen calx (weicher Kalkstein) und skr. carkara Kiesel (khara, hart, rauh) ist nur ein scheinbarer. Denn wenn Curtius calx zu carkara stellt, so denkt er dabei jedenfalls an die ursprüngliche Bedeutung des Wortes d. h. Stein schlechtweg, während die Bedeutung Kalkstein allem Anscheine nach erst die spätere ist. Auch $\chi \alpha^{\prime} \lambda \iota \xi$ ist zunächst Kiesel, erst später Kalk geworden, wie es scheint. Die Entlehnung von calx aus $\chi u^{\prime} \lambda \iota \xi$ ist so gut wie sicher, man vergleiche den noch vorhandenen Vokal auf einer Inschrift (nach Weise) aus dem Jahre 134 v. Chr.: basilicam calecandam. Paul. Fest. S. 47.4 calicata aedificia.

8. Die Eigenschaft und Kraft des Magneteisensteins ist schon lange vor Lucrez bekannt gewesen. Lucretius 6, 908: . . quem magneta vocant patrio de nomine Graii. Vergl. Plinius 36, 127: sideritim (= magnem) ob id alio nomine vocant, quidam Heraclion (= magnes). magnes appellatus est $a b$ inventore, ut auctor est Nicander- in Ida, ut reperio, namque et passim inveniuntur, in Hispania quoque; invenisse autem fertur clavis crepidarum baculi cuspide haerentibus, cum armenta pasceret. quinque genera magnetis Sotacus demonstrat: Aethiopicum et a Magnesia Macedoniae - - - et in Magnesia Asiae.

9. Namen von Edelsteinen finden sich bei römischen Schriftstellern erst seit dem 1. Jabrhundert v. Chr. beryllus

1) Durch griechischen Einfluss. Homer II, VI, 244, 248. gri $\lambda \alpha \mu \circ \iota$

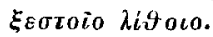

Journal f. prakt. Chemie [2] Pd. 61. 
530 Diergart: Etymologische Untersuchungen u.s. w.

kommt zuerst bei Properz vor. Vergl. Plinius 37, $76 \mathrm{f}$. India eos (berullos) gignit, ... .

10. Sulfur ist nach $W$ eise verwendet worden in vineis Plin. 17, 264, ad poliendas vestes Plin. 35, 198, ad conciunanda vina Plin. 14, 129.

Selbstredend kann die vorliegende Arbeit keinen Anspruch auf Vollständigkeit machen. Weitere Untersuchungen über die Ableitung der in der chemischen Wissenschaft und Technik gebräuchlichen Benennungen gedenke ich demnächst folgen zu lassen, und ich bitte die verehrten Fachgenossen, mir die Bearbeitung dieses Gebietes für einige Zeit freundlichst überlassen zu wollen.

Für die mir bereitwilligst ertheilten Rathschläge und Quellenhinweise in schwierigen Fällen sage ich folgenden Herren Universitätsprofessoren und Fachgelehrten meinen besten Dank:

A. Chwolles, W. Esch, S. Gabriel, M. Hartmann, E. Hübner, Ferd. Lindner, Erich Schmidt, E. Sieg, sämmtlich in Berlin.

\section{Verzeichniss der Abkürzungen.}

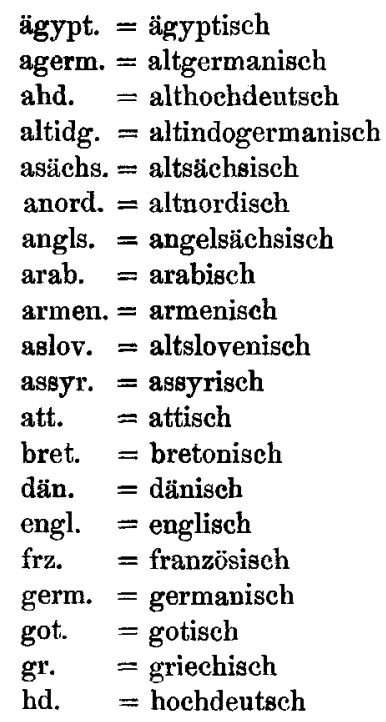




\section{Verzeichniss der benutzten Quellen.}

J. Chr. Adelung, magazin für die deutsche sprache, 2 Bde., Leipzig 1783; Georg Agricola, de animantibus subterraneis liber. Bas. 1549 , de natura fossilium libri $X$, Bermannus sive de re metallica dialogus; Becker, Die Sonne und die Planeten, Leipzig 1883; Benfey, Griechisches Wurzellexikon, Berlin 1842; Hu go Blüm ner, Terminologie und Technologie der Gewerbe und Künste bei Griechen und Römern, Leipzig 1887; Georg Curtiu s, Grundzüge der griechischen Etymologie, Leipzig 1866; Hermann Diels, Elementum, Leipzig 1899; Engel, Kypros, Berlin 1841; Fränkel, Die aramäischen Fremdwörter im Arabischen, Leyden 1886; Freytag, Arabisches Wörterbuch; Gesenius, Hebräisches Wörterbuch; Graham-Otto, Ausführliches Lebrbuch der Chemie, Braunschweig 1863; Jakob und Wilhelm Grimm, Deutsches Wörterbuch, Leipzig 1873; Valentin Hintner, Kleines Wörterbuch der lateinischen Etymologie, Brixen 1873; Jakob, Altarabisches Beduinenleben, Berlin 1897; John, Die Malerei der Alten; Kenngott, Handwörterbuch der Mineralogie, Geologie und Paläontologie (Encyklopädie der Naturwissensehaften), Breslau 1882; Fr. Kluge, Etymologisches Wörterbuch der deutschen Sprache, Strassburg 1899; Frauz von Kobell, Geschichte der Mineralogie von 1650-1860, München 1864; Herm. Kopp, Geschichte der Chemie, 4 Bde., Braunschweig 1843; J. G. Krünitz, ökonomisch-technologische encyklopädie, Berlin 1773; Camillus Leonardus, Speculum lapidum; Libavius, Alchymia; Albertus Magnus, De alchymia; Joh. Mathesius, bergpostilla oder Sarepta, Nürnberg 1578; Michael Merkati, Metallotheca; F. Max Müller, India what can it teach us? London 1883; Paracelsus, Traktat von Mineralien; Plinius, Historia naturalis; J. Ernst Rietz, ordbock öfver svenska allmoge spräket, häft 1, 2, Lund 1862; Oskar Schade, Altdeutsches Wörterbuch, 2. Aufl, Halle 1872-1882; Unger, Die Insel Cypern; Basilius Valentinus, Currus triumphalis antimonii; A1. Vanicek, Etymologisches Wörterbuch der lateinischen Sprache, Leipzig 1881; A. Weber, Indische Studien III: Oskar Weise, Die griechischen Wörter im Latein, Preisschrift der Fürstlich Jablonowskischen Gesellschaft, Leipzig 1882. 